\title{
Monitoring fibrinolytic activity in the cerebrospinal fluid after aneurysmal subarachnoid haemorrhage: a guide to the risk of rebleeding?
}

\author{
R S MAURICE-WILLIAMS Y B GORDON AND A SYKES
}

From the South East Thames Regional Neurosurgical Unit, The Brook Hospital, The Royal Free Hospital School of Medicine, and St. Bartholomew's Hospital, London

SUMMARY Serial assay of fibrin degradation products (FDPs) was used to monitor fibrinolytic activity in blood and cerebrospinal fluid (CSF) after subarachnoid haemorrhage (SAH) in 64 patients, 43 of whom had aneurysms. CSF levels fell rapidly from high initial values over a few days, thereafter more slowly to reach normal levels after one to six weeks. Probably only the later slow decline is a measure of fibrinolysis, which is obscured in the first few days by the cross antigenicity with FDP's of fibrinogen released by the bleed. After this phase of fibrinogen clearance, early attainment of normal FDP levels appears to be associated with a lower risk of rebleeding in cases of aneurysm. This phenomenon could be useful in deciding whether marginal cases should be treated surgically or conservatively.

During the last decade, attention has been focused on the possible tole of clot lysis in causing recurrent bleeding after an aneurysmal subarachnoid haemorrhage, and several reports have suggested that treatment with antifibrinolytic drugs may significantly reduce the incidence of such rebleeding. ${ }^{1-5}$ Relatively little work has been done however, to monitor the pattern of systemic and nervous system fibrinolytic activity after subarachnoid haemorrhage (SAH) and to investigate the way in which it is modified by antifibrinolytic therapy.

In this paper we report on the findings from a number of patients whose blood and cerebrospinal fluid (CSF) fibrinolytic activity after SAH were followed by serial assay of their fibrin and fibrinogen degradation products (FDP). Some of these patients received treatment with the antifibrinolytic drug tranexamic acid (TEA). The results suggest that it may be possible to isolate a group of patients whose risk of rebleeding is high despite such treatment.

Address for reprint requests: $M:$ RS Maurice-Williams, The Brook Hospital, Shooters Hill Road, London SE18 4LW

Accepted 14 July 1979

\section{Patients and methods}

Investigations were carried out on 64 patients. All were ctherwise fit persons who had had a single spontaneous subarachnoid haemorrhage confirmed by lumbar puncture, admitted to the neurosurgical unit of St. Bartholomew's Hospital, or to the South-East Thames Regional Neurosurgical Unit. All but two patients were admitted within 96 hours of haemorrhage and most had been relatively little affected by the bleed (57 were in Botterell Grades 1 or 2, that is, with no neurological deficit, slight to moderate meningism, alert or slightly drowsy, and six were in Botterell Grade 3, that is drowsy with a moderate focal deficit) ${ }^{6}$

Immediately after admission $2 \mathrm{ml}$, each of blood and CSF were taken and placed in plastic tubes containing $1 \mathrm{mg} / \mathrm{ml}$ of epsilonaminocaproic acid to suppress further fibrinolysis. After storage at $4^{\circ} \mathrm{C}$, the FDP's were estimated by the radioimmuncassay technique described by Gordon et al. ${ }^{7}$ This involves the use of highly purified fibrinogen degradation fragment $E$ for standardisation and Iodine ${ }^{125}$ labelling and a specific antiserum to the fragment. The radioimmunoassay sensitivity limit is $2 \mu \mathrm{g} / 1$., but for the present study the standard curve was targeted to 50 to $1000 \mu \mathrm{g} / 1$. The pre- 
cision as determined by the use of quality control sera showed an $8 \%$ variation during 18 months.

Further blood and CSF specimens were taken during the ensuing period up to a maximum of 49 days from admission. In general, they were obtained during the midpoint of the first week from admission, at the end of the first week and thereafter at weekly intervals. In those patients who required additional lumbar punctures for specific purposes (such as the treatment of communicating hydrocephalus), additional specimens of CSF were taken at those times. In patients given TEA, which was administered for a maximum period of 42 days, specimens were taken one to three days after cessation of treatment to investigate the possibility of rebound fibrinolysis. The number of paired blood and CSF specimens taken from individual patients is shown in table 1 .

Table 1 Number of paired samples taken from individual patients

\begin{tabular}{lll}
\hline No. of paired samples & All patients & Aneurysm patients \\
\hline $1-4$ & 29 & 13 \\
$5-12$ & 35 & 30 \\
\hline
\end{tabular}

The upper limit of the normal serum level of FDPs obtained by this method of assay was already known $\left(300 \mu \mathrm{g} / 1^{8}\right)$, but no previous CSF assays had been performed. Presumed normal CSF FDP levels were obtained by ascays of CSF removed from 14 patients with conditions which it might be reasonably assumed would probably not alter CSF levels. In these patients the diagnoses were: pituitary micro-adenomas 5 , trigeminal neuralgia 1 , headaches of probable functional origin 2 , skull vault tumour 2 , systemic myelofibrosis 1 , hypertension 1 , lumbar spondylosis 1 , cerebral atrophy 1 .

The apparent causes of the subarachnoid haemorrhage as established by cerebral angiography or autopsy in the 64 patients are shown in table 2 , together with the number who rebled during follow-up of four to 22 months.

Table 2 Cause of haemorrhage and number of patients who rebled

\begin{tabular}{lcc}
\hline & $\begin{array}{l}\text { Total No. of } \\
\text { patients }\end{array}$ & $\begin{array}{l}\text { No. who } \\
\text { rebled }\end{array}$ \\
\hline Aneurysm & 43 & 14 \\
No causative lesion found & 12 & 0 \\
Angioma & 5 & 0 \\
Moya Moya Disease & 1 & 0 \\
$\begin{array}{l}\text { Cause unknown (angiography or autopsy } \\
\text { refused) }\end{array}$ & 3 & 1 \\
\hline
\end{tabular}

Of the 43 patients with aneurysms, only 15 had intracranial operations, at a mean interval from the presenting haemorrhage of 21 days (span nine-57 days). FDP assays were stopped at the time of operation. A further three patients were treated by cervical ligation of the common carotid artery. Two of five angioma patients had intracranial operations at 20 and 25 days respectively.

In those patients given tranexamic acid, treatment began at the time of admission and continued for 42 days if they proved to have aneurysms deemed unsuitable for surgery. If surgery was performed, TEA was stopped at the time of operation, and if angiography showed no causative lesion, it was stopped after angiography. Overall 42 of 64 patients received TEA, including 30 of 43 of those with aneurysms. The drug was given at a daily dose of six $\mathrm{g}$, by continuous intravenous infusion for 4-7 days, thereafter orally in four divided doses.

\section{Results}

NORMAL CSF FDP LEVELS

Levels ranged between less than $100 \mu \mathrm{g} / 1$ (six patients) and $170 \mu \mathrm{g} / 1$. We have accordingly set the upper limit of normal as determined by this method as not more than $170 \mu \mathrm{g} / 1$.

\section{SERUM FDP LEVELS}

These remained within normal range after subarachnoid haemorrhage. Only two patients had raised levels (between 1100-2100 $\mu \mathrm{g} / \mathrm{l}$ ) for periods of 7-10 days. In one case this coincided with a leg deep venous thrombosis.

\section{CSF FDP LEVELS AFTER SUBARACHNOID}

HAEMORRHAGE FROM ANEURYSMS

In the accompanying figures, CSF FDP levels (in $\mu \mathrm{g} / 1)$ are plotted against time. CSF readings taken after rebleeding have been excluded. Aneurysm patients have been divided into the four groups as shown in table 3 .

Table 3 Aneurysm patients only:

\begin{tabular}{|c|c|c|c|}
\hline \multirow{2}{*}{ Given TEA } & Rebled & 8 & \\
\hline & No rebleed & 22 & \\
\hline \multirow{2}{*}{ Not given TEA } & Rebled & 6 & \\
\hline & No rebleed & 7 & \\
\hline
\end{tabular}

It will be seen that FDP levels are initially generally very high and fall rapidly during the 
first week, more slowly thereafter. It seems as if the FDP decline curve may be analysed into two components-an initial rapid decline over a few days superimposed on a more slow decay extending over several weeks. A possible reason for this is postulated in the discussion.

Although levels in the first week are generally very high they are not uniformly so and there is great variability.

There appears to be no relationship between initial levels and the likelihood of a rebleed. Those who rebled subsequently had a mean level (on Days 1 or 2) of $4310 \mu \mathrm{g} / 1$ as against 3820 $\mu \mathrm{g} / 1$ amongst those who did not. For patients not treated with TEA the figures are 3480 and 4030 respectively. The rate of decline of the CSF FDP levels in the first few days appears similar in all groups, whether or not they received TEA and whether or not they rebled. Separation out of the decline patterns during this period in individual cases has likewise shown no characteristic pattern permitting separation of rebleeders from non-rebleeders or any difference in the decline if TEA was given.

After the first few days, however, a difference in the decay curves becomes apparent. In those who received TEA, and who did not rebleed subsequently, normal levels are reached earlier and the later slope is more flattened than in the three other categories where the late decay pattern appears set at a higher level. The most striking difference appears to be amongst the TEA-treated patients, between those who did rebleed and those who did not.

Early attainment of a normal CSF FDP level appears associated with a reduced likelihood of rebleeding. In the table 4 , the numbers who had at least one level below $200 \mu \mathrm{g} / 1$ in the first 21 days after the bleed are shown for each category. It will be seen that of 13 patients who reached a normal level within 21 days, only one rebled. Of 30 patients who did not, 14 rebled.

Conversely, of 14 aneurysm patients who had rebled, only one had had a normal level (FDP less than $200 \mu \mathrm{g} / 1$ ) within 21 days, as opposed to 12 of 29 patients who did not rebleed ( $p<0.05$ by Fisher Exact Probability Test).

\section{CSF FDP LEVELS IN THOSE WITH NO CAUSATIVE LESION FOUND}

It appeared that decay to normal levels after the first few days is more marked in those given TEA (four of seven patients reached subnormal levels within 21 days as opposed to one of five), but it was felt that the data were insufficient to justify any firm conclusions.
Table 4

\begin{tabular}{|c|c|c|c|c|}
\hline \multirow{2}{*}{ Aneurysms: } & & \multirow{2}{*}{$\begin{array}{r}\text { Total } \\
22\end{array}$} & \multicolumn{2}{|c|}{$\begin{array}{c}C S F F D P 200 \mu g / l \\
\text { within } 21 \text { days }\end{array}$} \\
\hline & $\int$ no rebleed & & 9 & $(41 \%)$ \\
\hline & rebled & 8 & 0 & $(0 \%)$ \\
\hline \multirow{2}{*}{13 not given TEA } & Sno rebleed & 7 & 3 & $(43 \%)$ \\
\hline & rebled & 6 & 1 & $(16 \%)$ \\
\hline
\end{tabular}

CSF FDP LEVELS IN THOSE WITH ANGIOMAS

With only five patients (two received TEA, none has rebled) no valid conclusions could be drawn. However, three of these patients had unusually low initial levels (on Days one to three) of between 250 and $320 \mu \mathrm{g} / 1$.

PERSISTENCE OF RAISED CSF FDP LEVELS Raised CSF FDP levels persisted for variable lengths of time after a subarachnoid haemorrhage. Excluding levels taken after a rebleed, the numbers of patients who had readings taken after various days after the haemorrhage and the number of those who had elevated readings beyond these dates are shown in table 5 .

Table 5

\begin{tabular}{|c|c|c|}
\hline & $\begin{array}{l}\text { Number } \\
\text { of } \\
\text { cases }\end{array}$ & $\begin{array}{l}\text { Number of CSF } \\
F D P s>200 \mu G / 1 \\
\text { after that day }\end{array}$ \\
\hline $\begin{array}{l}\text { CSF levels obtained on Day } 14-20 \\
\text { CSF levels obtained on Day } 21-27 \\
\text { CSF levels obtained on Day } 28-34 \\
\text { CSF levels obtained on Day } 35 \text { or later }\end{array}$ & $\begin{array}{l}35 \\
25 \\
28 \\
15\end{array}$ & $\begin{array}{r}27 \\
20 \\
10 \\
7\end{array}$ \\
\hline
\end{tabular}

THE EFFECT OF DISCONTINUING TEA ON CSF FDP LEVELS

Sixteen patients had CSF FDPs measured on the day on which TEA was stopped and at a subsequent time within three days. The TEA was stopped at between eight and 46 days from the haemorrhage. In only one case was there a rise in the level at the subsequent reading. This was a patient is whom the TEA was stopped on Day 40. At this time the CSF FDP level was 210 $\mu \mathrm{g} / 1,48$ hours later it was $820 \mu \mathrm{g} / 1$.

\section{Discussion}

Immediately after a haemorrhage from an aneurysm the rebleed risk is small, but the daily risk then rises steadily to reach a peak between the 7 th and 10th Days. ${ }^{9}$ This may be a reflection of progressive lysis of the fibrin and red cell clot in and around the aneurysm. 
The initial haemorrhage activates the normally quiescent fibrinolytic system of the leptomeninges, and suppression of this process may account for the apparent efficacy of antifibrinolytic drugs in preventing early rebleeding. ${ }^{2}$ Antifibrinolytic drugs are widely used in the initial management of subarachnoid haemorrhage. Several controlled studies have reported reduced rebleeding from aneurysms when they are given. ${ }^{341011}$ Aminocaproic acid and tranexamic acid, the two drugs in current use, both act by competitively inhibiting the activation of plasminogen to plasmin. Experiments in both man and animals suggest that tranexamic acid is the more potent antifibrinolytic of the two. ${ }^{12}$ While antifibrinolytic drugs may reduce the rebleed rate, however, they do not abolish rebleeding altogether. Although clot lysis is probably only one of several factors concerned in the propensity of an aneurysm to rebleed, it seemed possible that monitoring fibrinolytic activity might be a guide to the risk of rebleeding in individual patients. For instance, it seemed conceivable that patients showing excessive fibrinolytic activity might be at especial risk.

The fibrinolytic activity of a body fluid can be measured in one of two ways-either by lysis of a fibrin plate in vitro, or by assay of the degradation products that are released by plasmic digestion of fibrin and fibrinogen. In the present study a highly sensitive and specific radioimmunoassay for fibrinogen degradation fragment $\mathrm{E}$ has been used. The radioimmunoassay is capable of accurately measuring small concentrations of fibrinogen degradation fragments and thus provides the basis for the early detection of fibrinogen and fibrinolysis.

After a SAH, both systemic and central nervous system fibrinolysis might be relevant, for the fibrin plug will be partly in the aneurysm sac, partly in the subarachnoid space.

Evidence for activation of the systemic fibrinolytic system after SAH is contradictory, though it is known that other stress-producing situations, such as mental stress, physical exertion and lumbar air encephalography ${ }^{13}$ lead to increased fibrinolytic activity of the blood. After air encephalography there is a rise in the fibrinolytic activity of both blood and CSF but with different time curves, and it has been suggested that different mechanisms are at work, possibly release of meningeal plasminogen activator and a general autonomic mechanism respectively. ${ }^{13}$ The present study showed uniformly low serum FDP levels at all intervals after a haemorrhage, even within 24 hours. Only two patients, one of whom had a concurrent systemic venous thrombosis, had elevated levels. This accords with the findings of Tovi et $a l,{ }^{13}$ who, using the immunochemical method of Niléhn, ${ }^{14}$ found no detectable FDPs in the blood, and only a modest early increase in blood fibrinolytic activity by the fibrin plate method. Also by direct methods, both Gibbs and $O^{\prime} G^{\prime}$ rman $^{15}$ and Ettinger ${ }^{16}$ found systemic fibrinolysis to be slightly increased after SAH. Watanabe $e t a l^{17}$ found an increase in blood FDPs, but they were using the insensitive and nonspecific latex coagulation test.

In the CSF, Levy and Silver ${ }^{18}$ found fibrinolytic activity with the fibrin plate technique three and 15 days after a SAH, but this finding could not be repeated by Tovi $^{2}$ who found weak activity using this method is only five out of 33 patients. However, Tovi did find high CSF FDP levels in every case. He explains this discrepancy by suggesting that although the release of plasminogen activator into the CSF after a haemorrhage is not enough to give lysis detectable by the fibrin plate method, yet continual lysis of the fibrin plug might give detectable CSF FDP levels. Alternatively, the FDP assay may have been detecting fibrinogen released into the CSF as a result of the initial bleed. On the other hand, Smith and Upchurch $^{19}$ found positive fibrinolysis in the CSF after SAH by the plate method, and this was reduced by the antifibrinolytic drug EACA.

The only previous systematic studies of CSF FDP levels after SAH are those of Tovi, ${ }^{2}$ who regarded the method as a reliable guide to nervous system fibrinolytic activity. He found CSF FDPs as early as three days after the bleed. A high initial level fell over the next few weeks, and by the end of the third week was often virtually undetectable. In four patients treated with TEA, the CSF FDPs fell steadily over a 4-hour period after 1g. of it had been given intravenously. After eight days of TEA, the CSF FDPs had disappeared, only to reappear after three weeks when the drug was stopped. In the current investigation, using a more specific and sensitive assay system for the detection of raised CSF FDPs, levels persisted in a number of patients five weeks after the bleed. This suggests that clot lysis might still be occurring. If this were so, then antifibrinolytic therapy should continue for at least as long as this if the aneurysm had not been operated on. It is unlikely that CSF FDPs would linger on for long after active clot lysis had ceased, as the total CSF volume is replaced every eight hours ${ }^{20}$ and FDPs have a biological half-life of 15 hours. ${ }^{21}$ 
Tovi's ${ }^{2}$ findings suggest that rebound fibrinolysis might occur after stopping antifibrinolytic therapy-in his case after 21 days. However, our findings do not support this, in that no rise in CSF FDPs occurred when the drug was discontinued at between eight and 46 days from the bleed, except in one case. It is possible, however, that a rapid rebound rise in CSF FDPs had already subsided by the time that the subsequent CSF specimen was taken, which was never less than 24 hours after the TEA had been stopped. Certainly there are theoretical reasons for supposing that rebound fibrinolysis might take place. $^{19}$ First, plasminogen might accumulate during treatment, to be suddenly converted to plasmin when treatment stopped, and second, both antifibrinolytics in general use have an enhancing effect on the action of plasmin when their concentrations are low.

It will be seen that the decay curves for CSF FDP levels with the passage of time appear to be composed of two superimposed slopes. An early slope falling steeply over a few days is superimposed on a more gradually declining slope which may linger on at above normal level for several weeks. Only the latter slope may be a reflection of release of FDPs by clot lysis. The earlier slope is probably due to cross antigenicity between fibrinogen and FDPs, and merely reflects the clearance of blood components from the CSF after a bleed. If so, then one would expect the initial rapid decay to be similar in all groups of patients and this is indeed so. It is only after the first few days that the element contributed by clot lysis would become dominant. At this stage, it can be seen that in one group, those patients given antifibrinolytics who did not rebleed, there is a tendency for normal CSF FDP levels to be achieved early on. Indeed, whether given TEA or not, rebleeding appears less likely if normal levels are reached within three weeks. Only one of 13 patients who did so rebled (nil of eight given TEA) as against 14 out of 30 patients who failed to reach normal levels within that time. This would be expected if continued fibrinolysis were a major factor in causing rebleeding.

If these findings are confirmed by further studies, CSF FDP assay could be of great value in the management of ruptured aneurysms. Because of the poor results of surgery in the first week, the recent tendency has been to defer operating on aneurysms until the second week or later, using antifibrinolytic cover during the waiting period. ${ }^{22}$ In those cases where surgery is likely to be particularly difficult or hazardous, knowing the risk of rebleeding might tip the balance for or against operation, particularly in view of recent evidence that antifibrinolysis alone may improve the long-term risk of rebleeding. ${ }^{3}$ It would seem that in patients given an antifibrinolytic who fail to reach normal CSF FDP levels early on, the risk of rebleeding is greater. This may be a reflection of insufficient suppression of clot lysis. Whatever the reason, however, surgery will be more strongly indicated than if normal levels had been achieved. Having said this it must be stressed that persistently high CSF levels do not mean that rebleeding is inevitable, nor do low levels mean that the risk of rebleeding can be discounted. It does seem that measurements are only useful once the first three to four days after the bleed have passed. This would limit the value of such an assay if very early surgery was being contemplated.

CSF FDP levels have been found to be sometimes raised in neurological conditions other than $\mathrm{SAH}{ }^{23}{ }^{24}$ Anderson et $\mathrm{al}^{24}$ found that FDPs were accompanied by other low molecular weight coagulation pnoteins but not the heavier ones. They believe that this indicates an origin from leakage across the blood-brain barrier and not from lysis within the subarachnoid space. However, there appear objections to this hypothesis. First, there is no relationship between blood and CSF FDP levels, a fact also noted by Hunter et $a .^{23}$ In addition, there was no tendency observed for high CSF FDP levels to be more persistent in more obtunded patients, as would have been expected were a leaking blood-brain barrier the source of the FDPs. One must conclude that clot lysis is indeed their substantial point of origin, that their decline (after the initial period) is an index of the control of fibrinolytic activity, and that they may prove a useful tool in deciding whether certain patients should be managed surgically or conservatively.

We would like to thank $\mathrm{Mr} \mathrm{R}$ Campbell Connolly for permission to carry out investigations on those patients in this study who were treated at St. Bartholomew's Hospital under his care.

\section{Figures}

The CSF levels (in $\mu \mathrm{g} / \mathrm{L}$ ) are plotted against the number of days since the haemorrhage. Readings from patients who subsequently rebled are shown is filled circles, those from patients who did not rebleed in open circles. All readings are prior to any rebleed. 


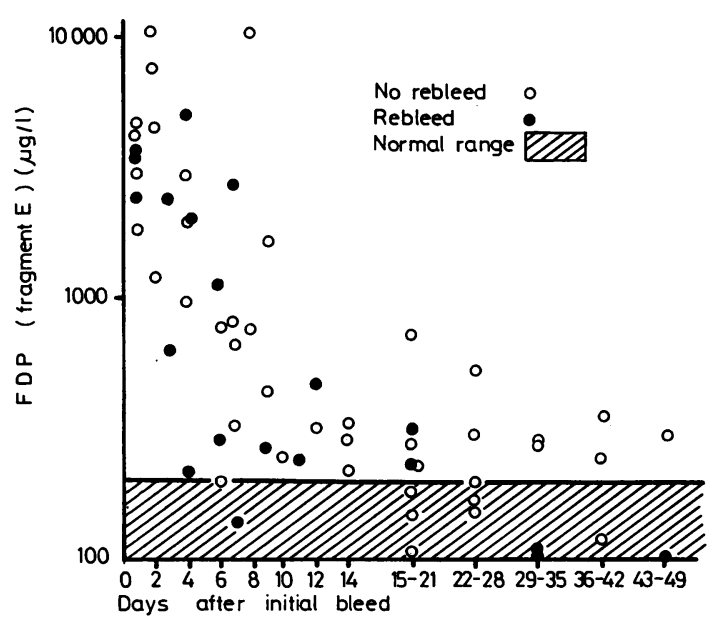

Fig 1 Patients not given antifibrinolytic treatment.

\section{References}

1 Corkhill G. Earlier operation and antifibrinolytic therapy in the management of aneurysmal subarachnoid haemorrhage. Med J A ust 1974; 1:468-70.

2 Tovi D. Studies on fibrinolysis in the Central Nervous System. Umea University Medical Dissertations N1.8, 1972.

3 Maurice-Williams RS. Prolonged antifibrinolysis: an effective non-surgical treatment for ruptured intracranial aneurysms? $B$ Med $J$ 1978; 1:945-7.

4 Sengupta RP, So SC, Ortega FJV. Use of E.A.C.A. in the pre-operative management of ruptured intracranial aneurysms. $J$ Neurosurg 1976; 44:479-84.

5 Ransohoff J, Goodgold A, Benjamin MV. Preoperative management of patients with ruptured intracranial aneurysms. J Neurosurg 1972; 36:525-30.

6 Botterell EM, Longheed WM, Scott JW, Vandewater SL. Hypothermia and interruption of carotid or vertebral circulation in the surgical management of intracranial aneurysms. J Neurosurg 1956; 13:1-42.

7 Gordon YB, Martin MJ, McNeile AT, Chard T. Specific and Sensitive Determination of fibrinogen degradation products by radioimmunoassay. Lancet 1973; 2:1168-70.

8 Gordon YB, Cooke ED, Bowcock SA, Ratky SM, Pilcher MF, Chard T. Non-invasive screening for venous thrombo-embolic disease. $B J$ Haematol 1977; 35:505-10.

9 Locksley HB. Natural History of subarachnoid haemorrhage, intracranial aneurysms and arteriovenous malformations. J Neurosurg 1966; 25:321-68.

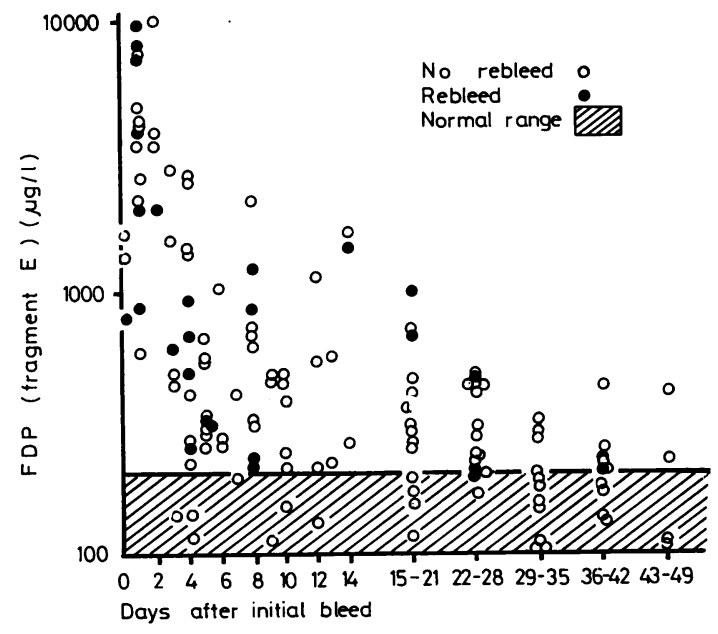

Fig 2 Patients given antifibrinolytic treatment (TEA).

10 Nibbelink DW, Torner JC, Henderson WG. Intracranial aneurysms and subarachnoid haemorrhage. Stroke 1975; 6:622-9.

11 Fodstad H, Liliequist B, Schannong M, Thulin CA. Tranexamic acid in the pre-operative management of ruptured intracranial aneurysms. Surg Neurol 1978; 10:9-15.

12 Anderson L, Nilsson IM, Niléhn JE, Hedner V, Granstrand B, Melander B. Experimental and clinical studies on AMCA. Scand J Haematol $1965 ; 2: 230-47$.

13 Tovi D, Nilsson IM, Thulin CA. Fibrinolytic activity of the cerebrospinal fluid after subarachnoid haemorrhage. Acta Neurol Scand 1973; 49:1-9.

14 Nilehn JE. Separation and estimation of "split products" of fibrinogen and fibrin in human serum. Thromb Haemostas 1967; 18:487-98.

15 Gibbs JR, O'Gorman P. Fibrinolysis in subarachnoid haemorrhage. Postgrad Med J 1967; 43:779-84

16 Ettinger MG. Coagulation abnormalities in subarachnoid haemorrhage. Stroke 1970; 1:139-42.

17 Watanabe $H$, Ito $M$, Chigasaki $H$, Ishii S. Antifibrinolytic therapy in ruptured intracranial aneurysm through repeated monitoring of fibrinolytic activity of blood. Neurol Med Chir 1976: 16:91-6.

18 Levy BJ, Silver D. The ability of epsilon amino caproic acid to cross the blood-brain barrier and reduce the spinal fluid fibrinolytic activity. Surg Forum 1968; 19:413-4.

19 Smith RR, Upchurch JJ. Monitoring antifibrinolytic therapy in subarachnoid haemorrhages. $J$ Neurosurg 1973; 38:339-44.

20 Milhorat. Hydrocephalus and the Cerebrospinal Fluid. Baltimore: Williams \& Wilkins, 1972. 
Monitoring fibrinolytic activity in cerebrospinal fluid after aneurysmal subarachnoid haemorrhage 181

21 Hardisty RM. In: Hardisty RM, Weatherall DJ, eds. Blood and its disorders. Oxford: Blackwell, 1974.

22 Mullan S. Current Controversies in Neurosurgery. Philadephia: W. B. Saunders, 1976: 259-69.

23 Hunter R, Thomson T, Reynolds CM, Pitcher P. Fibrin-fibrinogen degradation products in cere- brospinal fluid of patients admitted to a psychiatric unit. J Neurol Neurosurg Psychiatry 1974; 37:249-51.

24 Anderson M, Matthews KB, Stuart J. Coagulation and fibrinolytic activity of cerebrospinal fluid. J Clin Path 1978; 31:488-92. 